\title{
Conformation des bovins de race Nélore (Bos indicus) au ranch Kila-département de la Cuvette en République du Congo
}

\author{
DIMI NGATSE Silvère, OGNIKA Alexis Jonas, AKOUANGO Parisse \\ Laboratoire des ressources Animales et biodiversité de l'Ecole Nationale Supérieure d'Agronomie et de Foresterie \\ (ENSAF), Université Marien N'GOUABI \\ Auteur correspondant : DIMI NGATSE Silvère, Tél : +242069552074 \\ E-mail:dimingatsesilure@yahoo.fr
}

Mots clés : Nélore, Mensurations, Conformation, Bovin, Congo

Keywords: Nélore, Measurements, Conformation, Cattle, Congo

\section{$1 \quad$ RESUME}

L'objectif de l'étude a été d'apprécier la conformation et certaines mensurations de la race bovine Nélore dans le but de maitriser leurs aptitudes à la reproduction. Les animaux avaient trois (3) ans d'âge. Les mensurations et la conformation ont concernées 89 génisses et 28 taurillons en stade de reproduction, sur un troupeau de 2587 têtes. Les mensurations corporelles concernées ont été évaluées par la méthode traditionnelle à la toise et au mètre ruban. Le poids vif moyen des mâles a été de 418,97 $\mathrm{kg} \pm 25,84 \mathrm{~kg}$ et celui des femelles de $333,67 \pm 33,12 \mathrm{~kg}$. La robe régulière est blanche, uniforme avec des membres sombres aux extrémités chez les mâles. La conformation indique de bonnes aptitudes bouchères et les mâles sont de grande taille. La hauteur au garrot a été de 1,54 $\pm 0,01 \mathrm{~m}$ et 1,48 $\pm 0,03 \mathrm{~m}$ pour les mâles et les femelles respectivement. La longueur scapulo-ischiale a indiqué 1,89 $\pm 0,03$ m et $1,81 \pm 0,02$ pour les mâles et femelles respectivement. Le périmètre thoracique de $1,82 \pm$ $0.03 \mathrm{~m}$ et $1,72 \pm 0,03 \mathrm{~m}$ respectivement pour les mâles et femelles. La liaison corrélative du poids vif a été plus marquée avec la hauteur au garrot $(\mathrm{r}=0,926)$ qu'avec le périmètre thoracique $(r=0,81)$ et la longueur scapulo-ischiale $(r=0,730)$. Il est donc important que les éleveurs procèdent à l'étude des mensurations et de conformation des Nelores pour bien apprécier leurs aptitudes futures de reproductions dans une ferme.

\begin{abstract}
The objective of the study was to highlight the morphometric characteristics and conformation of the Nélore bovine breed in order to control their breeding. The animals were all about three (3) years old. The measurements and conformation involved 89 heifers and 28 breeding bulls from a herd of 2,587 heads. The body measurements concerned were evaluated by the traditional method of the measuring board and measuring tape. The average live weight of males was $418.97 \mathrm{~kg} \pm 25.84 \mathrm{~kg}$ and that of females $333.67 \pm 33.12 \mathrm{~kg}$. The Common coat colour is uniform white, with dark extremities in the males. The conformation indicates good beefing abilities and the males are large. The height at the withers was $1.54 \pm 0.01 \mathrm{~m}$ and $1.48 \pm 0.03 \mathrm{~m}$ for males and females respectively. The scapuloischial length of $1.89 \pm 0.03 \mathrm{~m}$ and $1.81 \pm 0.02$ for males and females respectively and thoracic perimeter of $1.82 \pm 0.03 \mathrm{~m}$ and $1.72 \pm 0.03 \mathrm{~m}$ respectively for males and females. The correlation of body weight was more marked with the height at the withers $(r=0.926)$
\end{abstract}


than with the thoracic perimeter $(r=0.81)$ and the scapuloischial length $(r=0.730)$. It is therefore important that the breeders study the measurements and conformation in order to appreciate the future breeding abilities of the animals on a farm.

\section{INTRODUCTION}

L'étude des mensurations linéaires et de la conformation permet d'apprécier et prédire les aptitudes à la production et à la reproduction chez les animaux d'élevage, et particulièrement chez les bovins. Le stade de reproduction pour les animaux d'élevage est un moment crucial permettant l'extériorisation des caractères pour s'affirmer comme "animal agricole". A ce stade les animaux développent leur potentiel génétique de croissance et de reproduction (Akouango et al, 2010). Le Congo, dans la dynamique d'améliorer la productivité des bovins N'Dama, a décidé d'importer les bovins de race Nélore en vue de les adapter aux conditions climatiques du Congo et ensuite les utiliser comme race amélioratrice. La Nélore une race à double usage dans son berceau d'origine, notamment pour le labour et l'attelage (Gaur et al., 2002). La Nélore provient de zébus de race Ongole, le nom Nélore vient du district Nellore de l'ancienne province de Madras, dans l'État d'Andra, situé sur la côte est de l'Inde, où les premiers animaux ont été expédiés au Brésil d'où l'appellation de Nélore. En 1936 le livre généalogique fut créé, la race a été améliorée et hautement sélectionnée et s'est adaptée au climat du Brésil (ABCZ, 2007). La race s'est adaptée en Amérique latine et, vers 1950, Le Brésil est devenu le plus grand éleveur de Nélore en l'exportant vers l'Argentine, le Paraguay, le Venezuela, l'Amérique centrale, le Mexique, les États-Unis et dans nombreux autres pays. Dans tous les endroits où la race Nélore a été importée, sa contribution a été remarquable, que ce soit en race pure ou en croissement avec des races locales. Les femelles Nélores ont un excellent instinct maternel. Sa carcasse donne, un bon rendement, à l'abattage (Souza et al., 2012). Cette race a été développée exclusivement pour la production de viande. Sa viande est savoureuse et moins grasse que celle des races européennes élevées dans des conditions analogues (Pereira Sune, 2005). La Nélore tolère les plantes fourragères de qualité médiocre plus efficacement que les races européennes. Cette tolérance à utiliser du fourrage de mauvaise qualité provient de son origine zébu (Albuquerque et al., 2007). La race Nélore possède des caractéristiques productives subtropicales intéressantes telles que la résistance au stress parasitaire et calorique couplées d'efficacité de production dans des conditions minimales de gestion. Aussi, la race Nélore a été largement étudiée dans son aire d'origine, qui est l'Inde et dans son aire de dispersion notamment en Amérique et a fait preuve d'une bonne extériorisation des performances zootechniques (Freneau et al, 2008). Ainsi, de 2012 à 2014, 4418 têtes bovines de race Nélore ont été importées du Brésil et transférées dans la partie septentrionale du pays dans le département de la Cuvette, au ranch Kila. L'apport du sang à un certain degré par la Nélore chez la N'Dama pourrait améliorer certains paramètres de production chez la N'Dama. Le maintien du niveau de sang de la Nélore à un certain pourcentage donné permettra d'améliorer la production de viande chez les produits croisés par rapport à leurs parents de race locale N’Dama. La disponibilité des terres, des ressources fourragères et d'une importante hydrographie dont le pays dispose, sont des atouts dans la possibilité d'augmenter la productivité numérique et pondérale du cheptel bovin et de faire face à la demande croissante de viande. La présente étude porte sur la maitrise des caractéristiques morpho biométriques des reproducteurs de la race Nélore ainsi que leur corrélation en tant que critère de sélection en vue de la mise en reproduction. 


\section{MATERIEL ET METHODES}

3.1 Milieu d'étude : Le ranch Kila est situé au nord du Congo Brazzaville, département de la Cuvette à $15 \mathrm{~km}$ de la sous-préfecture d'Oyo, sur l'axe Oyo-Obouya entre le village Bara et Opokagnia. Il est situé au Sud de l'équateur, entre $1^{\circ} 11^{\prime} 25,80^{\prime}$ ' de latitude Sud et $16^{\circ} 01^{\prime}$ 56,11" de longitude Est du méridien de Greenwich à $311 \mathrm{~m}$ d'altitude par rapport au niveau de la mer, au sud-est de la Cuvette congolaise. Le ranch Kila dispose de 1991,03 ha répartis sur 22 parcs dont 14 parcs avec pâturages améliorés sur 660,77 ha, 7 parcs avec pâturages inondés sur 166,60 ha, 8 parcs avec pâturages naturels sur 1174,46 ha, soit 1982 ha consacrés aux pâturages. Les animaux séjournent dans les pâturages où ils passent la nuit, le matin les bouviers font la ronde des parcs pour enregistrer les naissances, les décès, et les animaux malades.

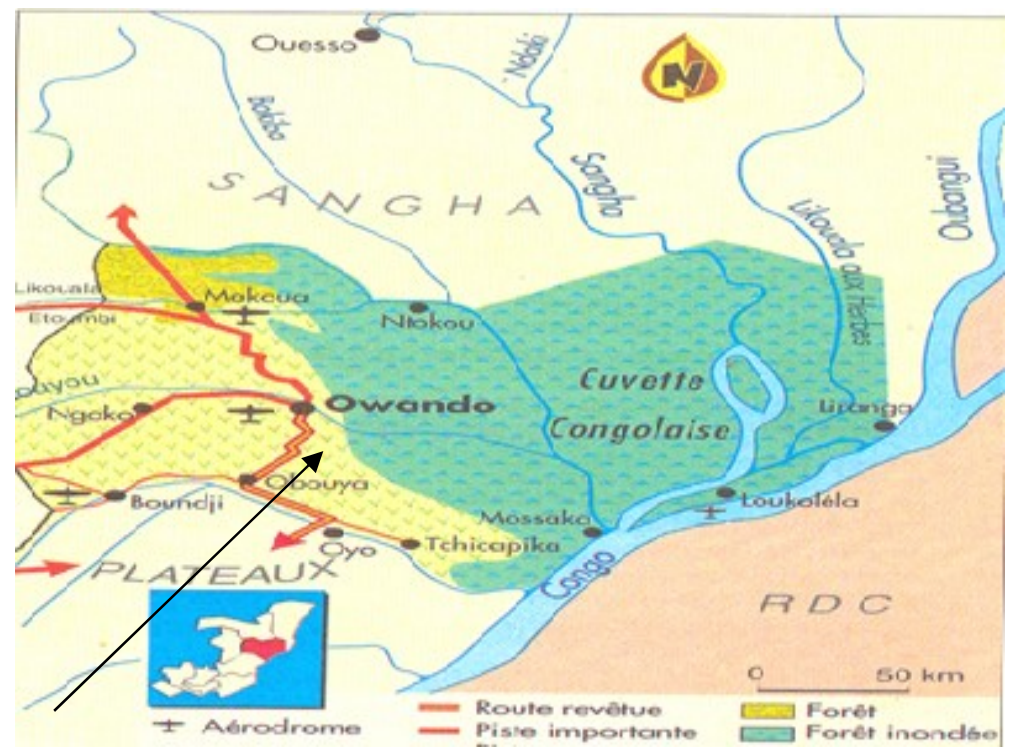

Figure 1: Ranch Kila -Département de la Cuvette (INRAP, 1999)

3.2 Aspects climatiques : La zone est sous l'influence du climat équatorial congolais de type "Guinéen Forest», caractérisé par une pluviométrie relativement importante, soit 1600 $-1800 \mathrm{~mm}$ d'eau par an, avec une température moyenne annuelle de $26^{\circ} \mathrm{C}$ et un écart thermique annuel faible de l'ordre de $2^{\circ} \mathrm{C}$. Dans cette localité, l'année se divise en quatre saisons : une grande saison des pluies d'octobre à décembre, une petite saison sèche de janvier à février, une petite saison des pluies de mars à avril et une grande saison sèche de mai à septembre (VENNETIER, 1977).

3.3 Aspects hydrographiques: La zone d'étude est arrosée principalement du côté Est par l'Alima, un des affluents du fleuve Congo, avec un débit moyen de $537 \mathrm{~m} 3 / \mathrm{S}$.
3.4 Aspects du sol et de la végétation : Dans la zone d'étude, le sol est caractérisé par une hydromorphie quasi permanente avec une accumulation de matières organiques. Il est sableux à hauteur de $86-96 \%$, riche en sable fin $(62-73 \%)$, en argile $(0-8,5 \%)$ et très perméable. Le $\mathrm{pH}$ oscille entre 5,2 et 5,9. L'humidité du sol varie également en fonction de la profondeur.

3.5 Troupeau : Le cheptel bovin du ranch Kila est composé essentiellement des animaux de race Nélore et se présente comme suit : 739 vaches, 748 génisses, 101 vêlles sevrées, 66 vêlles non sevrées. Pour les mâles, 31 taureaux, 651 taurillons, 138 veaux sevrés, 113 veaux non sevrés, soit un effectif total de 2587 animaux de 
la race Nélore. Notre étude a concerné 28 taurillons et 79 génisses.

3.6 Alimentation: Les animaux pâturent sur des parcours naturels. On trouve les espèces telles que : Bulbostylis laniceps, Trachypogon spicatus, Ctenium newtonii, Hyparrbenia wombaliensis (Mopoundza et al, 2016). La ferme a également crée des pâturages artificiels enherbés de: Panicum massaye, Brachiara brizenta, Brachiara omidicola. La distribution de la drèche de brasserie se fait deux (2) fois la semaine avec un complément minéral vitaminé à base de sel de cuisine. L'eau de boisson est servie ad libitum.

3.7 Prophylaxie : Les animaux sont soumis régulièrement à des soins de déparasitage et reçoivent un traitement à base de trypanocides. Ils sont vaccinés contre la pasteurellose et la péripneumonie contagieuse bovine et sont soumis au dépistage de la brucellose et de la tuberculose. Les montes sont naturelles, les taureaux reproducteurs y sont laissés en permanence.

3.8 Collecte des données : Les données relatives aux mensurations ont été collectées en utilisant la méthode traditionnelle de mesure à la toise et au ruban métrique. Ces méthodes demeurent encore dans les exploitations sous les tropiques malgré le risque de mesures directes et de grand temps consacré (Toszer et al, 2000). Les pesées ont été faites avec la balance automatique de marque COIMMA KM3-N, 4000kg de portée et $2 \mathrm{~kg}$ de précision. Le principe de mensurations est défini comme l'indique la figure 2 .

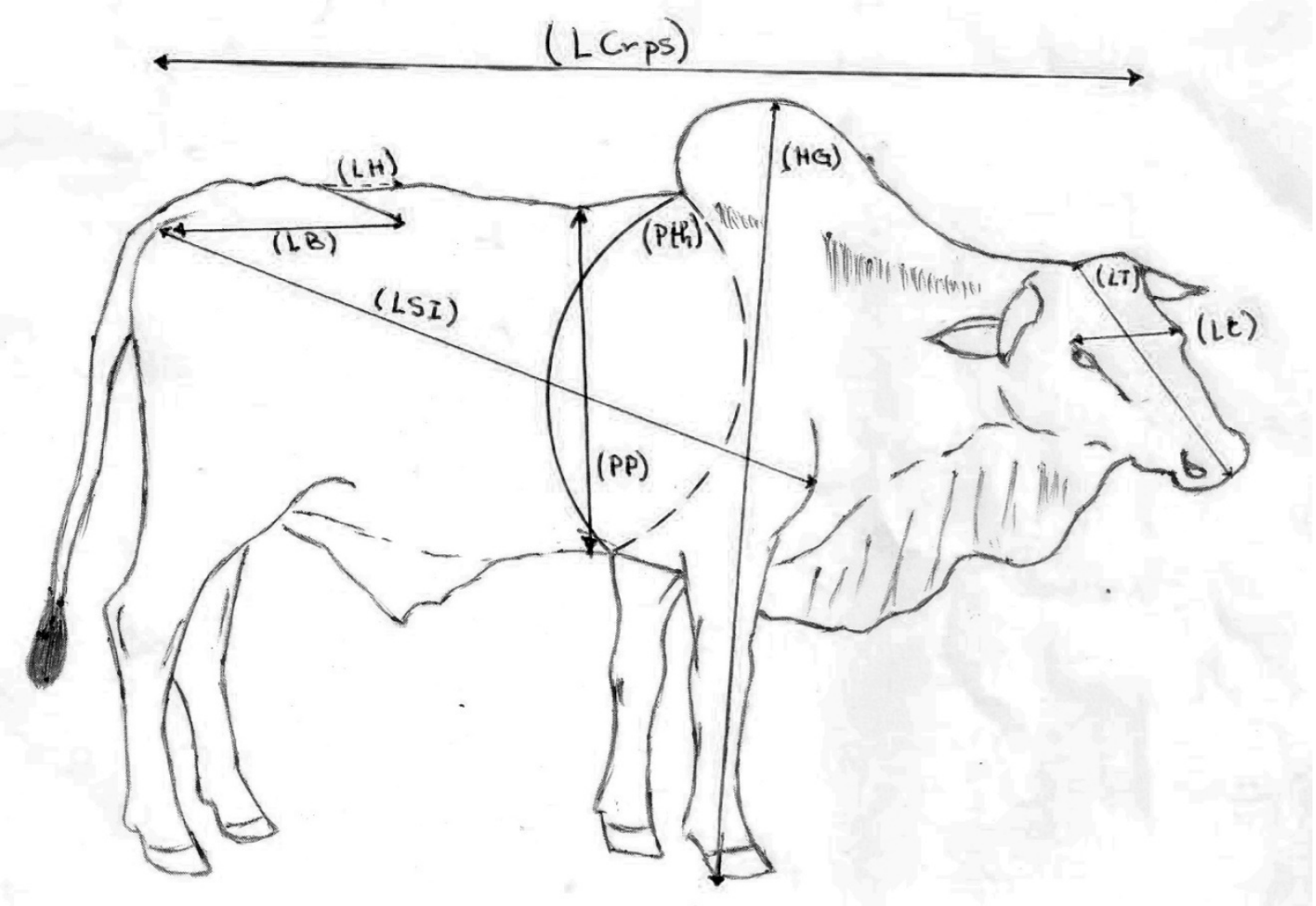

Figure 2 : Procédure des mensurations

(LT): Longueur de la tête ; Distance entre la nuque et le bout du nez;

(LCrps): Longueur du corps ; Distance entre la nuque et la pointe des fesses;

(LB): Longueur du bassin ; Distance entre les pointes des hanches et les pointes des fesses ;

(LSI): Longueur scapulo-ischial ; Longueur entre la pointe de l'épaule et la pointe de la fesse ;

(LH): Largeur aux hanches ; Distance entre les deux pointes des hanches ; 
(Lt) : Largeur de la tête ; Distance entre les deux (2) yeux ;

(Pth): Périmètre thoracique ; Mesure passant verticalement en arrière du garrot et au niveau du passage de sangle ;

(HG): Hauteur au garrot ; Distance entre le sommet du garrot et le sol ;

(PP): Profondeur de poitrine ; Estimé au passage de sangle à l'arrière de pattes antérieures.

Les mesures ont eu lieu dans la matinée avant l'abreuvement. L'âge des animaux a été rendu disponible grâce à la consultation des fiches individuelles et l'observation des boucles d'identification sur lesquelles sont inscrits le numéro du troupeau et le lot d'appartenance. Le mois de la naissance est marqué par l'encoche réalisée dans la bordure de l'oreille à l'aide d'un emporte-pièce. C'est leur localisation qui informe sur le mois de naissance. Quelques liaisons corrélatives ont été explorées

\section{RESULTATS}

4.1 Mensurations linéaires des bovins Nélores mâles et femelles: Le tableau 1 présente l'ensemble des résultats issus des différentes mesures corporelles effectuées sur les animaux Nélore mâles et femelles. Les poids vifs moyens ont été de 418,97£25,84 Kg et $333,67 \pm 33,12 \mathrm{~kg}$ respectivement pour les mâles entre les paramètres mesurés et le poids vif. Les données sur la conformation ont été explorées par observation directe des animaux et la consultation des fiches zootechniques retraçant la traçabilité de la race dans son aire d'origine et dans son aire de dispersion.

3.9 Analyse statistique : Les résultats obtenus ont été traités et présentés sous forme de moyennes et écart types, calculés et comparés avec le logiciel Excel.

et les femelles $(\mathrm{P} \leq 0,05)$. Chez tous les animaux, la hauteur au garrot $(1,54 \pm 0,01 \mathrm{~m})$, le périmètre thoracique $(1,82 \pm 0,03 \mathrm{~m})$ la longueur scapuloIschiale $(1,89 \pm 0,03 \mathrm{~m})$ et le poids vif moyen $(418,97 \pm 25,84 \mathrm{~kg})$ chez les mâles ont été plus grands que chez les femelles $(\mathrm{P} \leq 0,05)$.

Tableau 1 : Mensurations moyennes corporelles des bovins Nélores

\begin{tabular}{|l|l|l|}
\hline Mensurations & Mâles (N= 28) & Femelles (N= 79) \\
\hline Poids vifs (kg) & $418,97 \pm 25,84 \mathbf{a}$ & $333,67 \pm 33,12 \mathbf{b}$ \\
\hline Hauteur au garrot $(\mathrm{m})$ & $1,54 \pm 0,01 \mathbf{a}$ & $1,48 \pm 0,03 \mathbf{b}$ \\
\hline Périmètre thoracique $(\mathrm{m})$ & $1,82 \pm 0,03 \mathbf{a}$ & $1,72 \pm 0,03 \mathbf{b}$ \\
\hline Longueur scapulo-Ischiale $(\mathrm{m})$ & $1,89 \pm 0,03 \mathbf{a}$ & $1,81 \pm 0,02 \mathbf{b}$ \\
\hline Hauteur au passage des sangles (m) & $0,70 \pm 0,01$ & $0,69 \pm 0,01$ \\
\hline Longueur du corps (m) & $1,30 \pm 0,02$ & $1,21 \pm 0,03$ \\
\hline Longueur de la tête (m) & $0,69 \pm 0,02$ & $0,68 \pm 0,02$ \\
\hline Largeur de la tête (m) & $0,29 \pm 0,01$ & $0,28 \pm 0,02$ \\
\hline Longueur de la croupe (m) & $0,64 \pm 0,02$ & $0,59 \pm 0,02$ \\
\hline Largeur aux hanches (m) & $0,52 \pm 0,02$ & $0,47 \pm 0,03$ \\
\hline
\end{tabular}

Les lettres $\mathbf{a}$ et $\mathbf{b}$ désignent une différence statistiquement significative $(\mathrm{P} \leq 0,05)$, des critères concernés comparés entre mâle et femelle.

4.2 Corrélations entre le poids vif et quelques mesures linéaires : Les corrélations entre le poids vif et les mesures linéaires ont été positives chez tous les animaux. Elles ont toutes été positives dans les deux groupes. Cette liaison a été plus marquée avec la hauteur au garrot $(\mathrm{r}=0,926)$ et le périmètre thoracique 
$(\mathrm{r}=0,819)$ qu'avec la longueur scapulo-ischiale $(\mathrm{r}=0,730)$.

4.3 Conformation des bovins Nélores mâles : Les mâles Nélores élevés à la ferme Kila ont une conformation massive. Les mâles purs sont blancs, gris-blancs, ou froments, ayant à des endroits gris sur les épaules et sur la bosse s'étendant de l'avant jusqu'au cou. La ligne dorsale descend légèrement de la bosse au sacrum. La croupe est blanche et courte, la tête est longue et large avec un profil rectiligne ou légèrement convexe au visage. Les pattes sont larges bien développées, la queue est longue, touchant presque le sol. Les oreilles sont longues, larges et convexes, parfois pendantes. La bosse est bien placée sur le garrot à partir du dos avec une légère pente vers l'arrière, elle est en position thoracique. Le fanon et le fourreau sont proéminents ; le fanon est très bien développé et grandes en moyenne $34-39 \mathrm{~cm}$ de long, avec des plis du cou et du fourreau pendant chez les taureaux. Les cornes sont plus courtes chez les taureaux que chez les vaches en général. Elles se projettent dans une direction latérale ou vers le haut de la tête, elles peuvent être totalement absentes ou soit ovale en coupe transversale (figure 3).

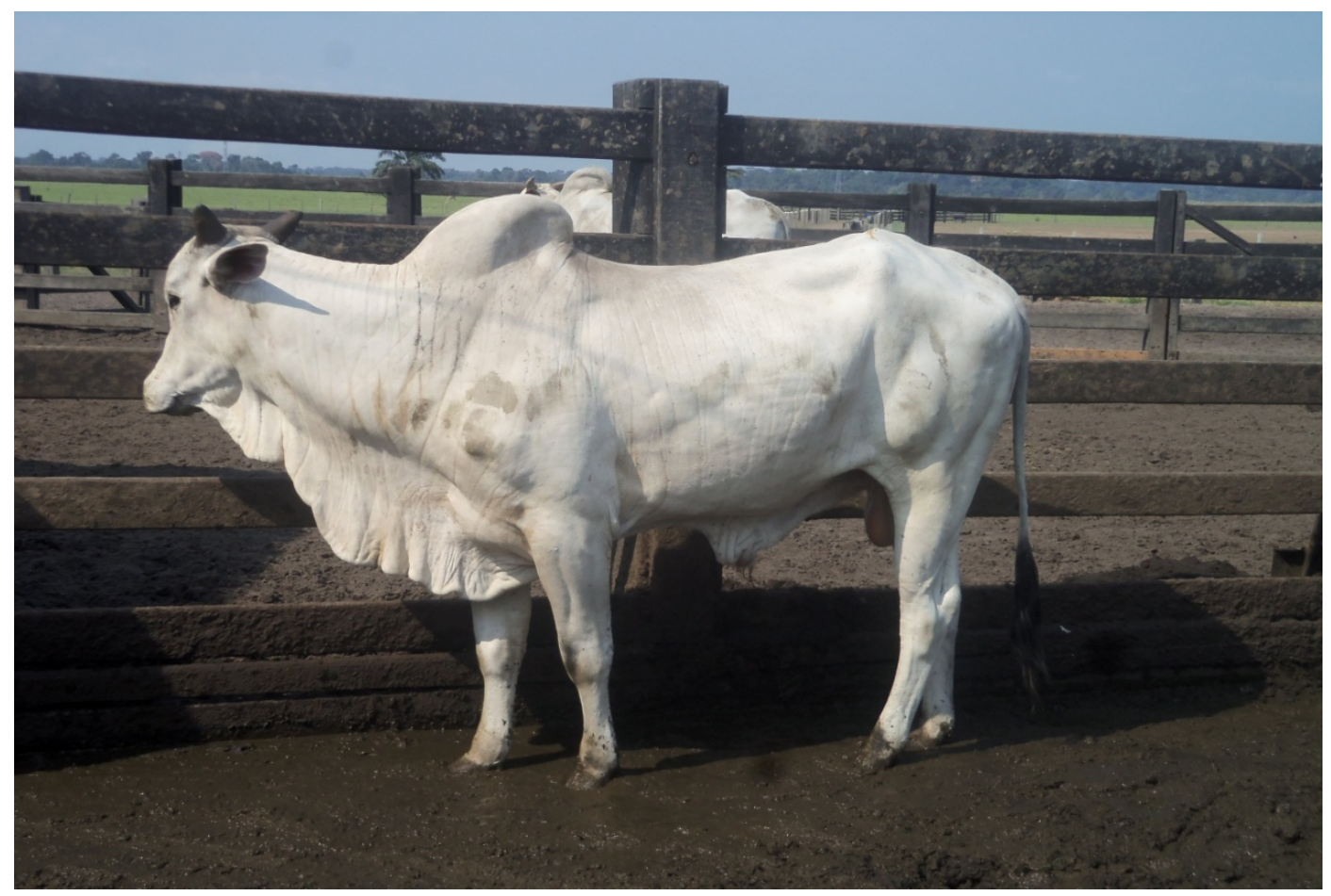

Figure 3 : Taurillon Nélore du ranch Kila

\subsection{Conformation Nélores femelles : Les} femelles Nélores élevées ont aussi une conformation massive. Les femelles sont blanches, parfois grises claires. Les cornes sont plus longues que les mâles et projetées vers le haut. La Ligne dorsale descend légèrement du cou au sacrum, elles sont sans bosse ; la croupe est blanche et courte ; les pattes sont courtes, les cuisses supérieures sont larges, bien développées que celles inférieures. La Queue est longue et bien développée, touchant presque le sol, les Oreilles longues, larges et convexes, parfois pendantes. La tête est longue et large, avec un profil rectiligne et parfois convexe au visage. Le pis est bien formé avec des tétines bien placées et bien développées (figure 4). 


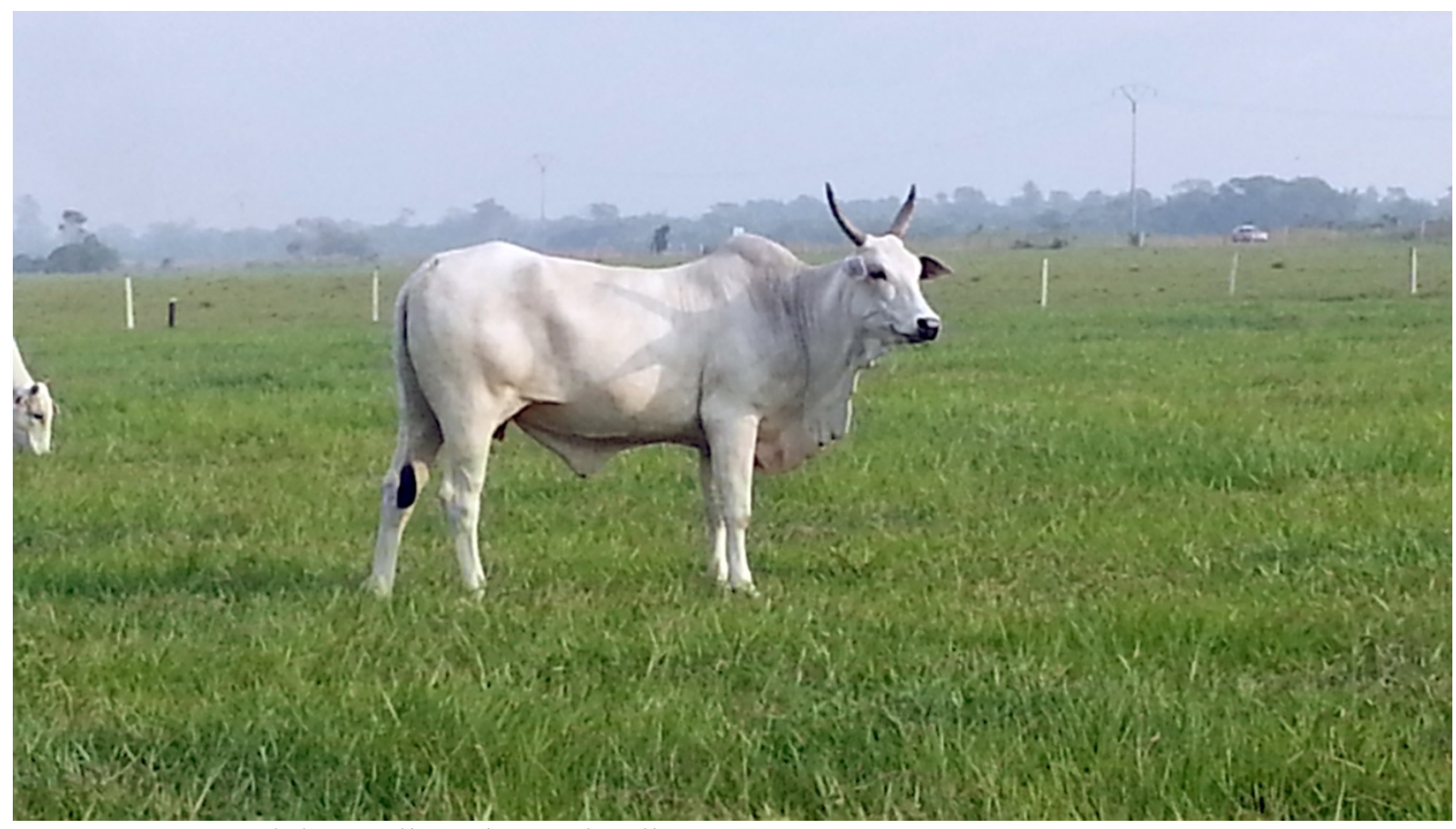

Figure 4 : Une génisse Nélore du ranch Kila

\section{DISCUSSION}

L'étude sur les mensurations linéaires et la conformation des bovins de la race Nélore élevés au ranch Kila a livré des informations intéressantes. Il sied de rappeler d'ores et déjà ici que le choix des reproducteurs mâles et femelles des Nélores s'opère à l'âge de trois (3) ans à la ferme kila; tandis qu'en Inde et au Brésil à cause de la prise de poids précoce des animaux, la mise en reproduction se fait à deux (2) ans. Le poids vif 498,8 $\pm 32 \mathrm{~kg}$ pour les mâles et $384,78 \pm 53,11 \mathrm{~kg}$ pour les femelles. Au ranch Kila les animaux ont présenté un seuil de poids de $418,97 \pm 25,84 \mathrm{~kg}$ et $333,67 \pm 33,12 \mathrm{~kg}$ respectivement pour les mâles et femelles à la reproduction à trois (3) ans. La prise de poids des Nélores à Kila est donc nettement très lente. Cela est dû aux conditions d'acclimatation, de conduite de l'alimentation dans les pâturages pauvres et semi inondés, dominés par du sable et l'eau. Dans cette étude,

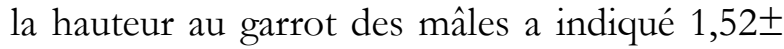
$0,01 \mathrm{~m}$ à 3 ans contre $1,40 \mathrm{~m}$ à 2 ans d'âge au Brésil, d'après les travaux de Freneau et al, (2008) et Blanchini et al, (2006). En Inde, Acharya (2005) travaillant sur les mêmes animaux dans leur berceau d'origine rapporte 1,65 $\mathrm{m}$ de hauteur au garrot chez les mâles ayant atteint la maturité. Ces différences peuvent être expliquées par la variation du système de sélection en fonction de l'âge et par la gestion des troupeaux exercée dans l'exploitation, et aussi par rapport aux conditions nutritionnelles et physiologiques subis par les animaux. Le Périmètre thoracique a été de 1,82 $\mathrm{m}$ cette valeur est supérieure à celle enregistrée par Acharya (2005) dans l'ordre de 171,4 m et inférieur a celle de ROCHA et al, (2003) qui est de 1,84 m. Ce paramètre étant lié fortement au poids traduit le mode d'alimentation avec complément ou non, la qualité du fourrage au sein de l'exploitation. La valeur de poids vif moyen des mâles est de 418, $97 \pm 25,84 \mathrm{~kg}$ et reste en dessous de celle obtenue par Kerst et al, (2017) qui ont travaillé sur les Nélores mâles de 17 mois d'âge indiquant 437,04 kg. Cela peut être expliqué par une bonne alimentation riche en protéine, et une supplémentation en minéraux adéquate, ce qui permet aux animaux d'extérioriser leurs performances de croissance et d'atteindre une 
maturité dans les délais. La hauteur au garrot des femelles donne 1,48 $\pm 0,03 \mathrm{~m}$, Bianchini et al (2006), ont enregistré $1,38 \mathrm{~m}$ et Acharya (2005) indiquent dans ses travaux 159,5 m. Le périmètre thoracique des femelles a été de 1,72 $\pm 0,03 \mathrm{~m}$. Freneau et al, (2008) indique la valeur similaire de 1,73 m sur les génisses de deux (2) ans. Sow et al, (1991), ont enregistrés $384,78 \mathrm{~kg}$ et $385 \mathrm{~kg}$ à 24 mois d'âge, tandis que celles de notre étude ont été de 333,67士 33,12 kg pour les femelles. Les espèces fourragères qui peuplent ces pâturages telles que Bulbostylis laniceps, Trachypogon spicatus, Ctenium newtonii, Hyparrbenia wombaliensis ont certainement une influence sur la croissance pondérale. Il faut rappeler aussi qu'il s'agit de la première génération des animaux Nélores nés au Congo, sans oublier la différence de température entre le Brésil et le Congo. Les mâles étudiés ont une bonne conformation. La robe dominante est blanche, mais on y trouve aussi la robe blanche avec des parties sombres sur le dos, les épaules, la bosse, le cou et au niveau des genoux. Ce qui peut être expliqué par différents croisements intervenus lors des différentes sélections naturelles. La Nélore est un animal très apte à produire la viande, une fois adapté au Congo avec une bonne alimentation elle est capable d'extérioriser ses performances. Les femelles aussi ont une conformation du corps qui indique de bonnes aptitudes bouchères. La robe dominante est blanche, elles n'ont pas de bosse. Quant aux corrélations entre le poids et les mesures linéaires, elles ont été toutes positives.

\section{REFERENCES BIBLIOGRAPHIQUE}

Acharya N.G. Seconde symposium international sur les bovins en cours 29-

31 octobre 2001 Rajendranagar, Hyderabad, Inde Université agricole de Ranga département d'élevage, GOVT. D'A.P. ASSOCIATION INDIENNE DES ELEVEURS DE BOVINS page 28.

Akouango F., Ngokaka F., Mopoundza C.P., Emmanuel K., 2010. Barymetrie formulas and control of growth of
Dans notre étude la hauteur au garrot et le poids a été la liaison la plus marquée. La variation des systèmes de sélection et le mode de gestion des troupeaux sont aussi des paramètres susceptibles d'influencer les résultats comme décrit dans les travaux de Freneau (2008). Par ailleurs, la grande capacité d'adaptation des Nélores aux conditions tropicales humides et aux fortes températures est connue (BrunA et al, 2014). Aussi, le choix de la race Nélore pour les besoins d'amélioration de la race N'Dama fait appel aux meilleurs critères de sélection des mâles et femelles. Le périmètre thoracique des animaux est fortement corrélé au poids vif, traduisant la relation intime entre les deux paramètres de croissance. Ce résultat peut être comparé aux travaux de Rocha et al, (2003), qui considère le périmètre thoracique comme la mesure linéaire de grande précision dans la détermination de la croissance des muscles. Cette dernière estime que les mesures de longueur scapulo-ischiale et de la hauteur ne conformation, indiquant clairement de bonnes aptitudes bouchères. Cette étude trouve son importance dans le projet d'amélioration de la race locale à partir de l'introduction des sujets Nélores dans le département de la Cuvette. Les élevages de ce département étant essentiellement peuplés de N'Dama, cette étude est essentielle pour les politiques de sélection des mâles et femelles de la race Nélore en. République du Congo.

breed cattle at Dihessé breeding farm in Congo Brazzaville. Pak. J. Nutr., 9 (5): 475-480, doi: 10, 3923/pjn. 2010. 475480.

Associação Brasileira Dos Criadores De ZebuABCZ. História. [2007] a. Disponível em:

$<$ http://www.abcz.org.br/site/abcz/hi storico.php>. Acesso em: 11/05/2019.

Bianchin, E.; Mcmanus, C.; Lucc, C. M.; Fernandes, M. C. B.; Prescott, E.; 
Mariante, A.S.; Egito , A .A. 2006. Características corporais associadas com a adaptação ao calor em bovinos naturalizados brasileiros. Pesquisa Agropecuária Brasileira, v. 41, n. 9, 2006.

Bruna L. R., Correlaçoes entre medidas corporais e caracteristicas das carcaça de tourinhos NÉLORE treminados em confinamentos. B. Indústr. Anim., Nova Odessa, v.71, n.4 p.371-380, 2014.

G. A. Norman Et P. E. De Felicio, « Effects of breed and nutrition on the productive traits of beef cattle in South-East Brazil - Part 1 \& 3 », Meat Science, vol. 5, p. 425-438, 1981.

G.K. Gaur, S.N. Kaushik \& R.C. Garg Ongole cattle status in India Project Directorate on Cattle, PH-7, Pallavpuram Phase 2, Modipuram, Meerut- 250 110, Uttar Pradesh, India

Gustavo Eduardo Freneau, Joao Carlos Cardoso Da Silva, Arcadio De Los Reys Borjas, Cristiane Amorim. 2008. estudo de medidas corporais, peso vivo e condiçao corporal de fêmeas da raça NÉLORE Bos taurus indicus ao longo de dose meses. Ciência Animal Brasileira, v. 9, n. 1, p. 76-85, jan. /mar. 2008

G.K. Gaur, S.N. Kaushik \& R.C. Garg. Ongole cattle status in India Project Directorate on Cattle, PH-7, Pallavpuram Phase 2, Modipuram, Meerut- 250 110, Uttar Pradesh, India

INRAP. - Géographie au CM CONGO. EDICEF Bleuzen F92178 VANVES, 1995, p. 50.

P. Akouango, P. Mopoundza, R. Pea. Ewomango, 2014. Étude des mensurations des bovins de race Ndama (Bos taurus) dans les pâturages naturels semi inondés de la ferme d'Abo au Congo Brazzaville. Journal of Animal \&Plant Sciences, Vol.20, Issue 3: $3137-$ 3143. ISSN 2071-7024
Paul Mopoundza, Silvère Dimi Ngatse, Alexis Jonas Ognika., Parisse Akouango. Caractérisation des pâturages naturels semi-inondés du département de la Cuvette pour un élevage bovin productif en République du Congo. Journal of Animal \&Plant Sciences, 2016. Vol.28, Issue 2: 4398-4408. ISSN 2071-7024

Rocha, E.D.; Andrade, V.J.; Euclides Filho, K.; Nogueira, E.; Figueiredo, G.R. 2003. Tamanho de vacas Nélore adultas e seus efeitos no sistema de produção de gado de corte. Arquivo Brasileiro de Medicina Veterinária e Zootecnia, v.55, p.474-479, 2003.

Rodrigo Sella Kerst, Daniel Augusto Da Silva, Fernanda Alves De Britto, Wilmar Sachetin Marcal, 2017. 8Biometria testicular como parâmetro selectivo de touros Nélore Revista Brasileira de Higiene e Sanidade Animal V. 11, n.2 (2017), ISSN: 1981-2965.

Sousa, G.G.T, GUSMÃO, F. B, LOPES, J. S. Características reprodutivas de bovinos da raça Nélore do meio Norte do Brasil. PUBVET, Londrina, V. 6, N. 21, Ed. 208, Art. 1390, 2012.

Sow. R. S, Denis J.P., Trail J.C.M., Thiongane P.J., Mbaye M. 1991.Notes sur la barymétrie comme moyen de sélection indirecte du poids vif. Rev. Elev. Med. Vet. Pays'Trop.44: 97-99.

Toszer J., Bedo S. 2000. Estimer les mensurations corporelles des bovins par analyse d'image vidéo. Rev.Rech. Ruminants; 7: 139P.

Vennier P. - « Le climat de la République populaire du Congo »In : Atlas de la République populaire du Congo. Ėdition jeune Afrique, 1977, pp.10-14.

Winker, K (1993) Rétrécissement de spécimens chez la paruline du Tennessee et Flycatchers de «Traill». J. Field Orn.64 :331-336.

Y. B. Pereira Suné, « Uma análise da comercialização de bovinos para abate 
no estado do Rio Grande do Sul », Universidade federal do Rio Grande do Sul, Porto Alegre, mémoire de master en zootechnie, 123 p., 2005. 